\title{
Circulating MicroRNA Profiles Differ between Hyperglycemia and Euglycemia in Coronary Heart Disease Patients
}

\author{
Yunyao Jiang, ${ }^{1,2,3}$ Nan Liu, ${ }^{1,2,4}$ Bingjie Xue, ${ }^{1,2,3}$ Jincai Hou, ${ }^{3}$ Chengren Lin, ${ }^{1,2}$ \\ Jianxun Ren, ${ }^{1,2}$ and Jianxun Liu, ${ }^{1,2}$ \\ ${ }^{1}$ Institute of Basic Medical Sciences, Xiyuan Hospital, China Academy of Chinese Medical Sciences, Beijing 100091, China \\ ${ }^{2}$ Beijing Key Laboratory of TCM Pharmacology, Xiyuan Hospital, China Academy of Chinese Medical Sciences, Beijing 100091, China \\ ${ }^{3}$ Jing-Jin-Ji Joint Innovation Pharmaceutical (Beijing) Co., Ltd., Beijing 100083, China \\ ${ }^{4}$ Beijing University of Chinese Medicine, Beijing 10029, China
}

Correspondence should be addressed to Jianxun Liu; jianxun_liu@163.com

Received 9 June 2017; Revised 25 September 2017; Accepted 1 October 2017; Published 30 October 2017

Academic Editor: Claudio Bucolo

Copyright (C) 2017 Yunyao Jiang et al. This is an open access article distributed under the Creative Commons Attribution License, which permits unrestricted use, distribution, and reproduction in any medium, provided the original work is properly cited.

\begin{abstract}
Coronary heart disease (CHD) has become one of the leading causes of death and functional impairment in the world. Hyperglycemia is associated with an increased risk of cardiovascular disease. It was speculated that miRNAs in peripheral blood were a primary parameter in discriminating CHD. The biological characteristics of coronary heart disease with hyperglycemia (HCHD) and coronary heart disease with euglycemia (ECHD) were investigated in the study. Circulating miRNAs from $26 \mathrm{HCHD}$ patients and 42 ECHD patients were identified by microarrays. Compared with the healthy patients, 15 and 20 differentially expressed miRNAs were identified in HCHD and ECHD groups, respectively. Gene ontology analysis was carried out by DAVID and functional annotations of the miRNA targets related to ATP binding, cellular components, protein binding, RNA binding, DNA binding, and so on. KEGG database was used for pathway analysis. Eleven pathways were identified in both HCHD and ECHD groups. Furthermore, 13 and 3 pathways were only identified in HCHD or ECHD group, respectively. And then, miRNA-gene regulatory networks were constructed to study the relationship between differentially expressed miRNAs and genes. This suggested that hsa-let-7c-5p and hsa-miR-24-3p might have the most important function for hyperglycemia in coronary heart disease patients.
\end{abstract}

\section{Introduction}

Coronary heart disease (CHD) is caused by atherosclerosis and thrombosis or atherothrombosis and has become one of the leading causes of death and functional impairment in the world $[1,2]$. It is reported that CHD may kill approximately seven million people and result in 129 million losses of disability-adjusted life years (DALYs) annually [3]. Studies reveal that more than $20 \%$ of patients with $\mathrm{CHD}$ have diabetes, about $35 \%$ are obese, about $45 \%$ have metabolic syndrome, more than $50 \%$ have hypertension, and almost $60 \%$ have dyslipidemia [4]. Hyperglycemia, not only in diabetic but also in the nondiabetic range, is associated with an increased risk of cardiovascular disease. This has been demonstrated by several trials and evidences that glycemic control appears to be linked to a reduced coronary artery disease [5]. Several mechanisms that hyperglycemia exacerbate myocardial damage and worsen the prognosis in patients with acute coronary syndrome have been revealed. Hyperglycemia results in myocardial cellular injury by producing free radicals and then inducing oxidative stress. In addition, hyperglycemia can promote osmotic diuresis and lower the circulating volume to affect cardiac contractility and decrease both end-diastolic volume and stroke volume [6].

MicroRNAs (miRNAs) are short endogenous and singlestranded noncoding RNA molecules that bind to the $3^{\prime}$ UTR of their target messenger RNA (mRNA) and inhibit or decrease translation of the mRNA into protein to regulate target gene expression $[7,8]$. MiRNAs regulate approximately $60 \%$ of human protein-coding genes and perform their functions in a number of biological processes such as cell development, differentiation, metabolism, immunity, apoptosis, 
and proliferation, which is conducive to many physiological and pathological conditions including cardiovascular diseases $[9,10]$. Atherosclerosis of the coronary arteries is a very complex process and is responsible for the development and clinical manifestations of CHD. The role of miRNAs in the process of manifestations has been evaluated through many researches and it is now recognized that miRNAs are connected to nearly all steps of atherogenesis, including beneficial and detrimental effects such as endothelial damage and dysfunction, monocyte-wall invasion and activation, lipoprotein formation, and platelet and vascular smooth muscle cell function exerting [11]. MiRNAs can give rise to degradation or blockade of protein translation because of negative regulation to the gene expression by binding to target mRNAs. MiRNAs can bind to target mRNAs to regulate the gene expression and lead to degradation or blockade of protein translation. In the circulation, they are resistant to degradation by being packaged as microvesicles, bound to protein complexes, or bound to high-density lipoproteins [12]. Therefore, miRNAs can be easily measured in circulation and the levels of miRNAs have been used as potential blood biomarkers for various pathologies. MiRNAs have been used as biomarkers in cancer diagnosis by detecting circulating levels of specific miRNAs, which have been associated with the pathogenesis of obesity, diabetes mellitus, and coronary artery disease and used in the diagnosis of myocardial infarction [13].

The circulating miRNAs are believed to come from circulating tumor cells and blood cells or other tissue cells affected by disease also directly release miRNAs into the blood stream [14]. Turchinovich et al. reported the theory of cell-cell communication via extracellular miRNA [15]. And they described the evidence that the short distance communication of cells was realized with extracellular miRNA. The long-range communication between different intercellular sites is also realized through the current models for the functionality of circulating miRNAs [16].

It was speculated that miRNAs in peripheral blood were a primary parameter in discriminating $\mathrm{CHD}$. The biological characteristics of CHD with hyperglycemia (HCHD) and CHD with euglycemia (ECHD) were researched by investigating circulating miRNA profiles and bioinformatics of HCHD and ECHD patients. And the common or different mechanisms between HCHD and ECHD were analyzed by gene ontology $(\mathrm{GO})$ and pathway analysis of miRNA targets. Functional annotations of the miRNA targets are related to transcription, cellular components, protein binding, RNA binding, and DNA binding, and so on. Twelve pathways, including p53 signaling pathway, cell cycle, FoxO signaling pathway, viral carcinogenesis, and chronic myeloid leukemia, were identified in both HCHD and ECHD groups. Furthermore, 13 and 3 pathways were only identified in HCHD or ECHD group, respectively.

\section{Materials and Methods}

2.1. Participant Recruitment. Ethical approval for this study was obtained and all procedures in this study were approved by the Medical Ethical Committee of Xiyuan Hospital of China Academy of Chinese Medical Sciences. A total of 87 volunteers were recruited from Xiyuan Hospital of China Academy of Chinese Medical Sciences. Among them, 19 healthy volunteers (HV) reported no CHD and exhibited a normal syndrome as judged by doctors. Of the 68 patients, 26 fulfilled study inclusion criteria for HCHD group and 42 fulfilled study inclusion criteria for ECHD group. The clinical studies followed the Helsinki declaration guidelines. Written informed consent was obtained from all volunteers.

2.2. Serum Sample Collection and Handling. Venous blood was obtained from each volunteer. Ten milliliters of venous blood was collected from ulnar vein and transferred into a vacuum blood collection tube. Samples were placed immediately in ice for blood coagulation and were centrifuged at $3000 \mathrm{rpm}$ for $20 \mathrm{~min}$ at $4^{\circ} \mathrm{C}$ to obtain serum. The obtained serum was taken to the study laboratory within 10 minutes.

2.3. RNA Isolation and Amplification. Total RNA including miRNA was isolated from the serum samples using Trizol reagent (Invitrogen, Canada) and purified using RNeasy Mini Kit (Qiagen, German) according to the protocol provided by manufacturers. It is ensured that slow molecular weight RNA can be retained in the purification. RNA concentration was determined by measuring the absorbance at $260 \mathrm{~nm}$ and quality control standard was $A$ (absorbance) ${ }_{260} / A_{280}=1.8-2.1$, using NanoDrop 2000 spectrophotometer (Thermo Fisher Scientific, USA).

2.4. Affymetrix miRNA Microarray. RNA was labeled with FlashTag Biotin HSR RNA labeling kit according to the manufacturer's instructions (Genisphere, USA). The Labeled RNAs were then hybridized to the Affymetrix GeneChip miRNA 3.0 arrays on the basis of the user manuals. Affymetrix Expression Console Software (version 1.3.1) was used to analyze the arrays, including data normalization, summarization, and quality control assessment. Median-centric normalization was used for the custom miRNA oligonucleotide chips and Affymetrix chips were normalized with the robust multichip analysis (RMA) procedure. Differentially expressed miRNAs were identified based on RVM $t$-test analysis. $p$ values were adjusted and corrected according to the Benjamini-Hochberg procedure and FDR (false discovery rate) $<0.05$ was chosen as the cutoff criteria. Differentially expressed miRNAs were considered to be upregulated or downregulated with at least 1.5-fold change in either direction with FDR (adjusted $p$ value $<0.05$.

2.5. Target Prediction of miRNA and Bioinformatic Analysis. The potential human miRNA targets were predicted with Tarbase (version 7.0) [17] and miRTarBase 2016 [18]. And the intersection set of these 2 databases was used for bioinformatic analysis. In order to investigate the biological process, cellular component, and molecular function of differentially expressed miRNA targets, GO analysis was carried out using DAVID [19] Bioinformatics Resources 6.8 databases. Functional categories were enriched within genes 
$($ FDR $<0.05)$ and the top $10 \mathrm{GO}$ functional categories were selected. DAVID that assigned Kyoto Encyclopedia of Genes and Genomes (KEGG) database was used for pathway analysis. The pathway analysis may discover a relation that was not easily visible from the changes of individual genes. The involvement of coexpressed genes in biological pathways was determined by pathway analysis. Pathways that had significant changes of FDR $<0.05$ were identified for further analysis. And the genes that significantly regulated pathways were selected for miRNA-gene regulatory network analysis. MiRNA-gene regulatory networks were constructed to study the relationship between differentially expressed miRNAs and genes in the context of biological pathways [20]. The miRNA-gene regulatory networks were constructed by Cytoscape 3.5.1 software [21] and betweenness centrality was used for topological analysis.

\section{Results}

3.1. Identification of miRNAs. MiRNAs from HCHD and ECHD patients were identified by microarrays. Compared with the healthy patients, 15 miRNAs with significantly differential expression (FDR adjusted $p$ value $<0.05$ and fold change $>1.5$ ) were identified in HCHD group (Table 1). Among them, 13 miRNAs were overexpressed and 2 were underexpressed. Twenty differentially expressed miRNAs were detected in ECHD group relative to HV, namely, 19 upregulated miRNAs and 1 downregulated miRNA (Table 2). Unsupervised hierarchic clustering was carried out based on the differentially expressed miRNAs and displayed as heat maps (Figure 1). The differentially expressed miRNAs overlapping and nonoverlapping between HCHD and ECHD groups were presented in Figure 2. Thirteen differentially expressed miRNAs that were upregulated were detected in both HCHD and ECHD patients (Figure 2(a)). One downregulated miRNA was significantly differentially expressed between ECHD and HV groups. And the downregulated miRNA was also differentially expressed miRNA in HCHD group (Figure 2(b)).

3.2. Gene Ontology (GO) Analysis. Gene ontology of miRNA targets was analyzed based on biological process, cellular component, and molecular function. The overlapping and nonoverlapping functional annotations between HCHD and ECHD groups were shown in Venn diagrams (Figure 3). One hundred and twenty-two GO terms and $141 \mathrm{GO}$ terms were significantly regulated (FDR $<0.05$ ) by the differentially expressed miRNAs in HCHD group and ECHD group, respectively. Among them, 73 upregulated (Figure 3(a)) and 23 downregulated (Figure 3(b)) functional annotations targeted by the differentially expressed miRNAs were indicated in both HCHD and ECHD groups. Top 10 significant upregulated and downregulated functional annotations of differentially expressed miRNAs in HCHD and ECHD groups were presented in Figures 4 and 5. In HCHD group, the highly enriched GO terms in biological process (Figure 4(a)), cellular component (Figure 4(b)), and molecular function (Figure $4(\mathrm{c})$ ) included viral process, protein stabilization,
TABLE 1: Thirteen overexpressed and 2 underexpressed circulating miRNAs of HCHD patients.

\begin{tabular}{lccc}
\hline Systematic name & FDR adjusted $p$ value & Fold change & Regulation \\
\hline hsa-miR-24-3p & 0.0257 & 1.5438 & $\mathrm{Up}$ \\
hsa-miR-26a-5p & 0.0257 & 1.5757 & $\mathrm{Up}$ \\
hsa-let-7c-5p & 0.0211 & 1.7598 & $\mathrm{Up}$ \\
hsa-miR-107 & 0.0333 & 1.8457 & $\mathrm{Up}$ \\
hsa-miR-92a-3p & 0.0292 & 1.8829 & $\mathrm{Up}$ \\
hsa-miR-103a-3p & 0.0331 & 1.9849 & $\mathrm{Up}$ \\
hsa-miR-25-3p & 0.0257 & 2.0408 & $\mathrm{Up}$ \\
hsa-miR-93-5p & 0.0302 & 2.1112 & $\mathrm{Up}$ \\
hsa-miR-106a-5p & 0.0331 & 2.1919 & $\mathrm{Up}$ \\
hsa-miR-191-5p & 0.0454 & 2.2617 & $\mathrm{Up}$ \\
hsa-miR-17-5p & 0.0211 & 2.3144 & $\mathrm{Up}$ \\
hsa-miR-16-5p & 0.0211 & 2.3306 & $\mathrm{Up}$ \\
hsa-miR-23a-3p & 0.0257 & 2.4398 & $\mathrm{Up}$ \\
hsa-miR-122-5p & 0.0412 & 1.5832 & Down \\
hsa-miR-320a & 0.0467 & 2.2958 & Down \\
\hline
\end{tabular}

TABLE 2: Nineteen overexpressed miRNAs and 1 underexpressed circulating miRNA of ECHD patients.

\begin{tabular}{lccc}
\hline Systematic name & FDR adjusted $p$ value & Fold change & Regulation \\
\hline hsa-miR-23b-3p & 0.0329 & 1.5214 & $\mathrm{Up}$ \\
hsa-miR-19b-3p & 0.0235 & 1.5383 & $\mathrm{Up}$ \\
hsa-miR-26a-5p & 0.0304 & 1.5392 & $\mathrm{Up}$ \\
hsa-miR-22-3p & 0.0304 & 1.6269 & $\mathrm{Up}$ \\
hsa-let-7b-5p & 0.0101 & 1.6539 & $\mathrm{Up}$ \\
hsa-miR-24-3p & 0.0304 & 2.0320 & $\mathrm{Up}$ \\
hsa-miR-103a-3p & 0.0327 & 2.1242 & $\mathrm{Up}$ \\
hsa-miR-107 & 0.0327 & 2.1653 & $\mathrm{Up}$ \\
hsa-miR-92a-3p & 0.0326 & 2.1859 & $\mathrm{Up}$ \\
hsa-miR-451a & 0.0479 & 2.2234 & $\mathrm{Up}$ \\
hsa-miR-25-3p & 0.0304 & 2.3831 & $\mathrm{Up}$ \\
hsa-let-7c-5p & 0.0104 & 2.5818 & $\mathrm{Up}$ \\
hsa-miR-17-5p & 0.0205 & 2.5950 & $\mathrm{Up}$ \\
hsa-miR-93-5p & 0.0327 & 2.6229 & $\mathrm{Up}$ \\
hsa-miR-191-5p & 0.0393 & 2.6341 & $\mathrm{Up}$ \\
hsa-miR-106a-5p & 0.0327 & 2.6382 & $\mathrm{Up}$ \\
hsa-miR-425-5p & 0.0473 & 2.7734 & $\mathrm{Up}$ \\
hsa-miR-23a-3p & 0.0304 & 2.8259 & $\mathrm{Up}$ \\
hsa-miR-16-5p & 0.0205 & 3.0602 & $\mathrm{Up}$ \\
hsa-miR-320a & 0.0409 & 1.9496 & Down \\
\hline
\end{tabular}

membrane, cytoplasm, ubiquitin-protein ligase binding, and ATP binding. As shown in Figure 5, the significant upregulated and downregulated functional annotations of differentially expressed miRNAs in ECHD group were cellcell adhesion, translational initiation, nucleoplasm, nucleus, protein binding, and poly(A) RNA binding.

3.3. Pathway Analysis. The pathways significantly (FDR < 0.05 ) affected by differentially expressed miRNAs were identified using KEGG pathway analysis. Eleven upregulated 


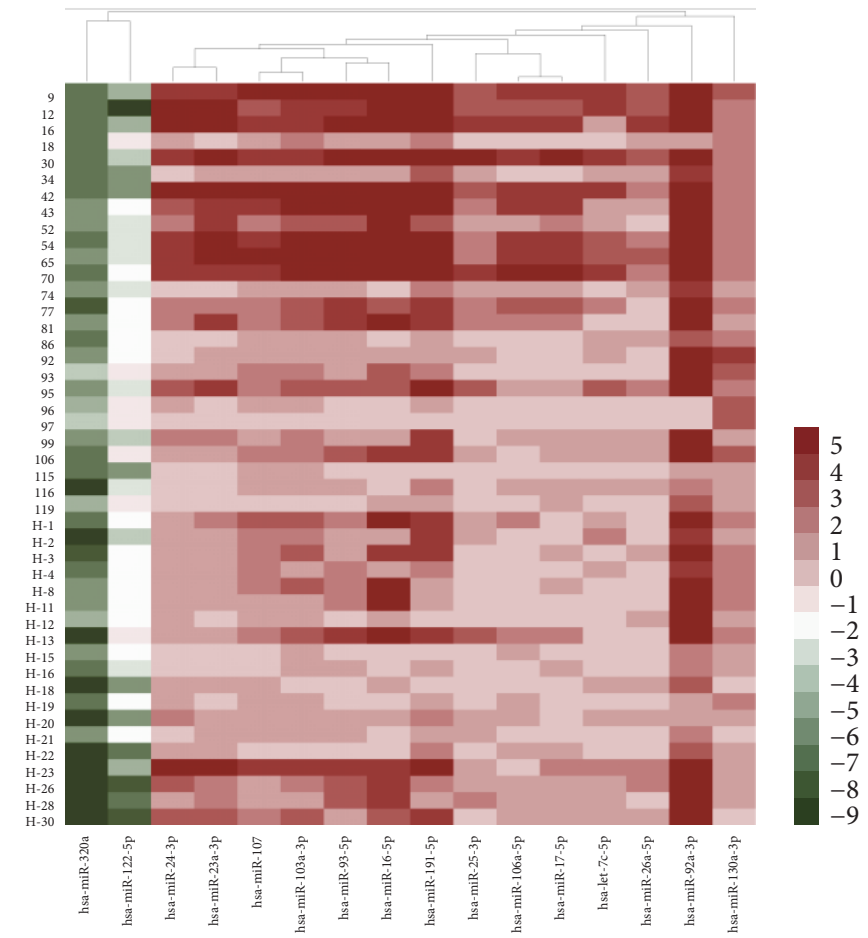

(a)

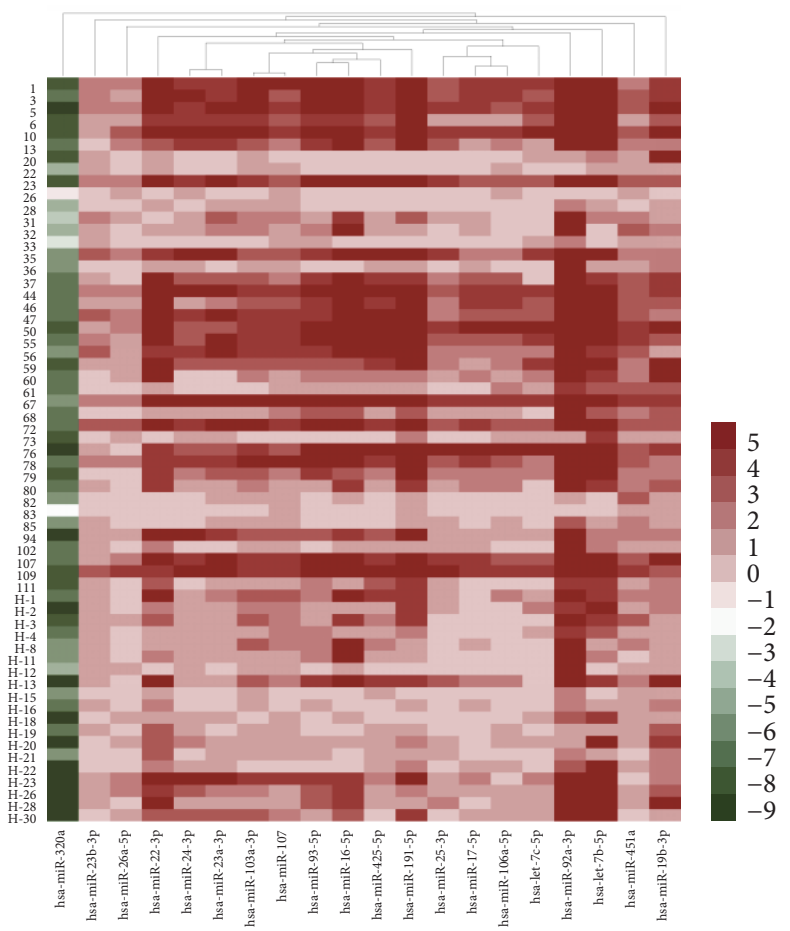

(b)

FIGURE 1: Cluster analysis of the differentially expressed microRNAs in the plasma compared between the HCHD patients and the healthy volunteers (a) or the ECHD patients and the healthy volunteers (b).

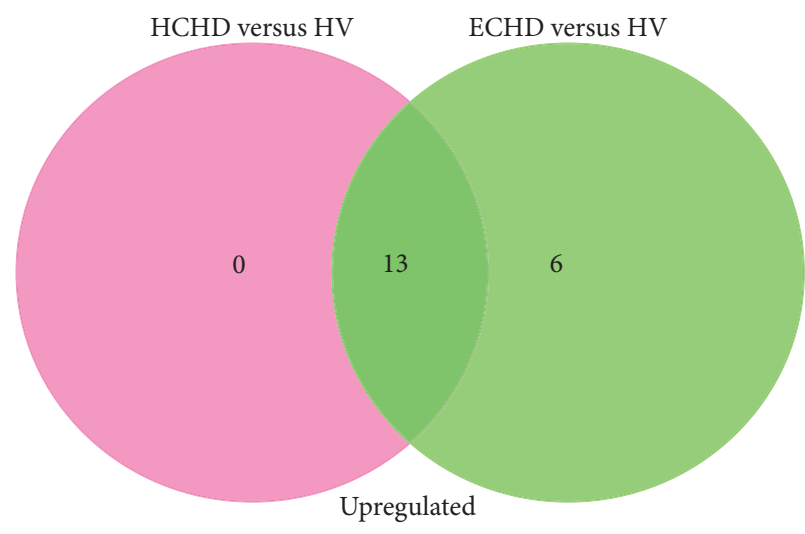

(a)

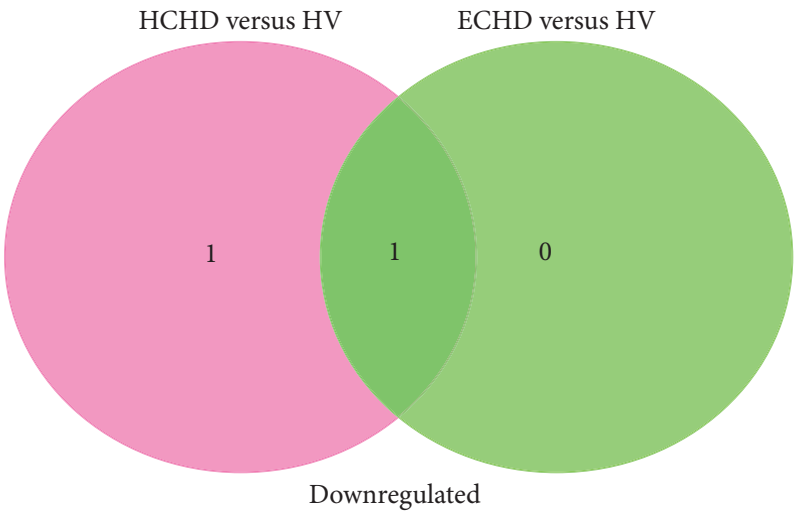

(b)

FIGURE 2: Venn diagrams of differentially upregulated microRNAs (a) and differentially downregulated miRNAs (b) in HCHD and ECHD patients.

pathways (Figure 6(a)) that included cell cycle, viral carcinogenesis, chronic myeloid leukemia, and p53 signaling pathway were identified in both HCHD and ECHD groups. And 1 downregulated overlapping pathway (Figure 6(b)) was shared by both HCHD and ECHD groups. Furthermore, 12 upregulated pathways and 1 downregulated pathway, including hippo signaling pathway, signaling pathways regulating pluripotency of stem cells, PI3K-Akt signaling pathway, and TGF-beta signaling pathway, were only identified in HCHD group. And the top 10 significantly upregulated pathways and one significantly downregulated pathway were shown in Table 3. Three specific pathways were potentially affected by the differentially expressed miRNAs in ECHD group. The significantly upregulated pathways were presented in Table 4.

3.4. miRNA-Gene Regulatory Network Analysis. The miRNAgene regulatory networks based on the genes that significantly regulated pathways were used to identify the putative target genes of the upregulated and downregulated miRNAs, which was shown in Figure 7. The betweenness centrality was 


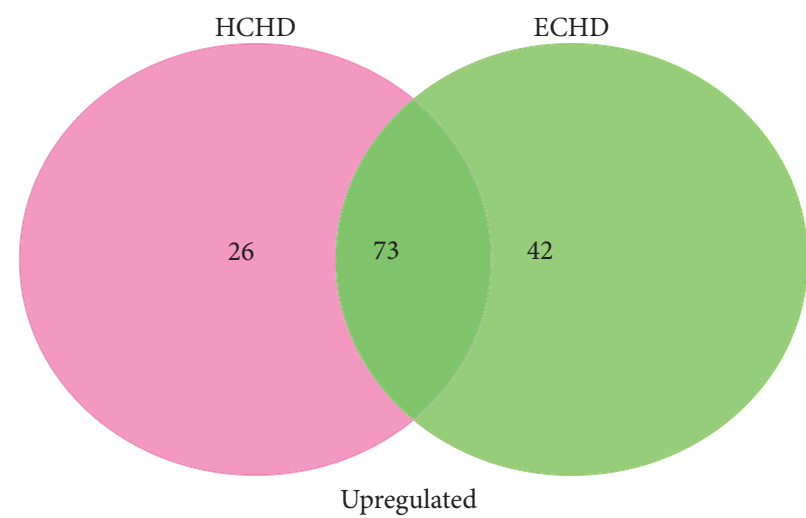

(a)

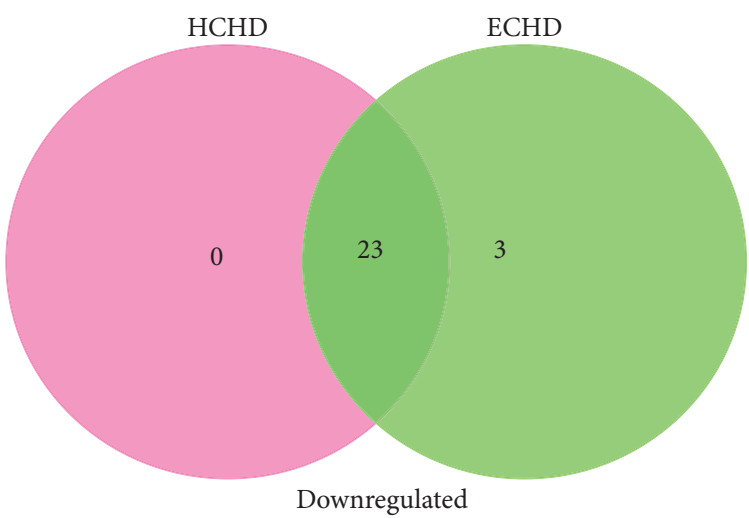

(b)

Figure 3: Venn diagrams of upregulated functional annotations (a) and downregulated functional annotations (b) from the target genes of differentially expressed miRNAs in HCHD and ECHD patients.

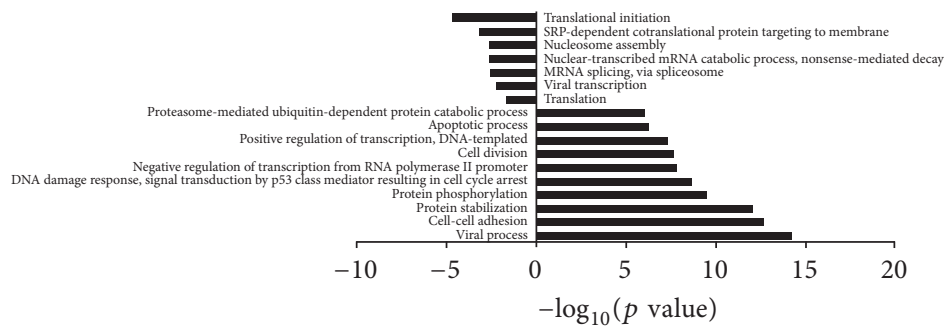

(a)

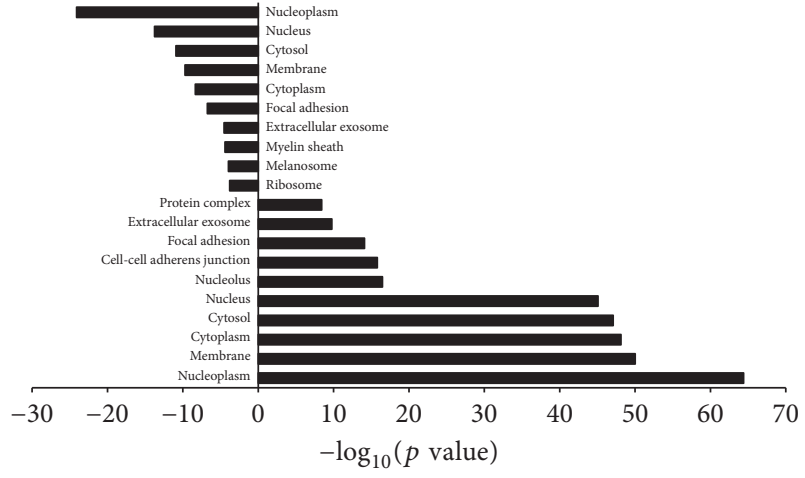

(b)

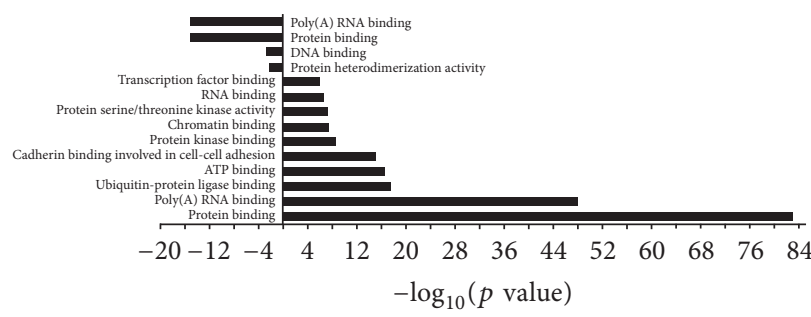

(c)

FIGURE 4: Significant upregulated and downregulated functional annotations of differentially expressed miRNAs in HCHD group. GO analysis according to biological process (a), cellular component (b), and molecular function (c), respectively, ranked by enrichment score $\left(-\log _{10}(p\right.$ value)).

used for topological analysis and the circles represented target genes and the squares represented miRNAs in the networks. In HCHD group, the total number of genes and miRNAs in the network was 272 and 15, respectively (Figure 7(a)). And the network diagram suggested the core miRNAs, namely, hsa-miR-16-5p, hsa-miR-17-5p, hsa-let-7c-5p, and hsa-miR24-3p, and their targets. Four dysregulated miRNAs, namely, hsa-miR-16-5p, hsa-miR-92a-3p, hsa-miR-93-5p, and hsamiR-17-5p, were core miRNAs in EHCD group (Figure 7(b)).

\section{Discussion}

The present study revealed differentially expressed circulating miRNAs between healthy volunteers and HCHD patients or ECHD patients. MiRNAs play an integral part in identifying the occurrence and development of various diseases and may be used as a new form of potential biomarkers [22]. Indeed, they are absolutely vital for the development of tissue and relevant to the pathological processes of numerous 


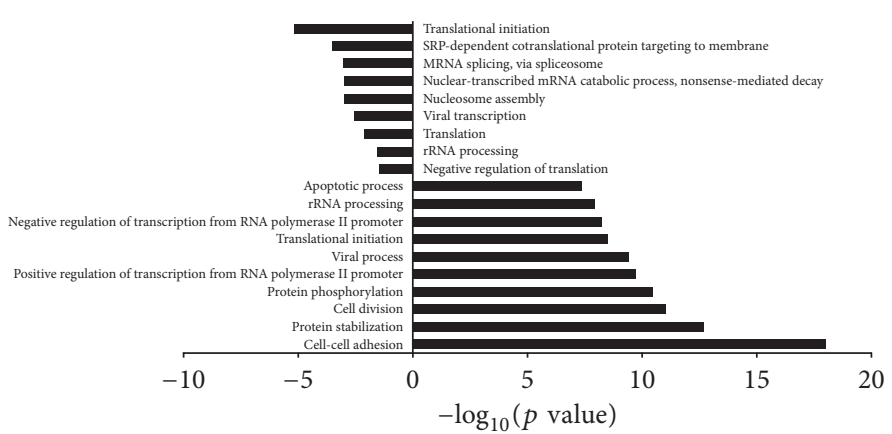

(a)

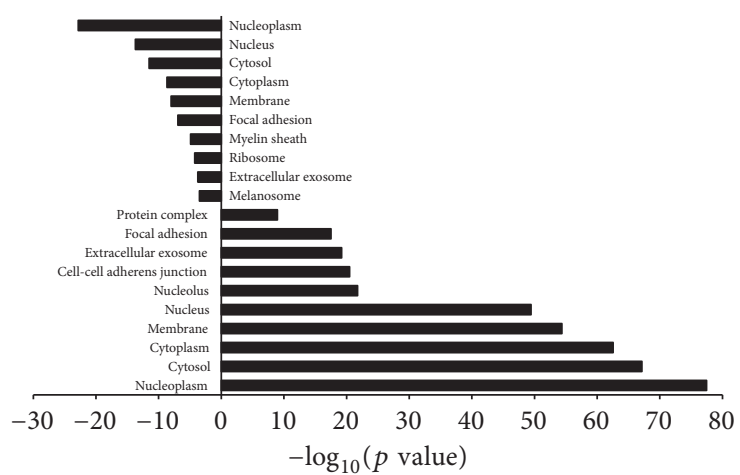

(b)

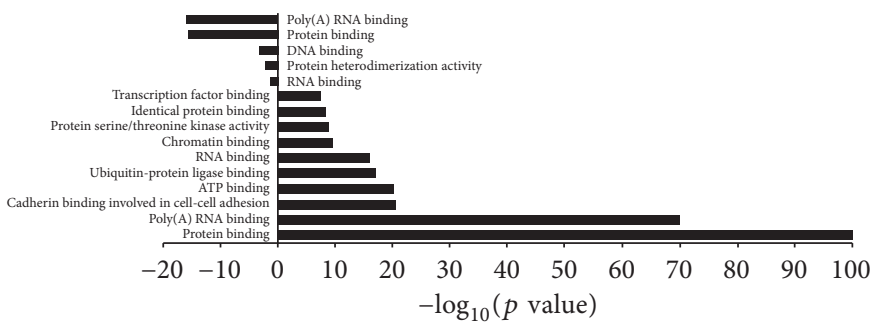

(c)

FIGURE 5: Significant upregulated and downregulated functional annotations of differentially expressed miRNAs in HCHD group. GO analysis according to biological process (a), cellular component (b), and molecular function (c), respectively, ranked by enrichment score $\left(-\log _{10}(p\right.$ value)).

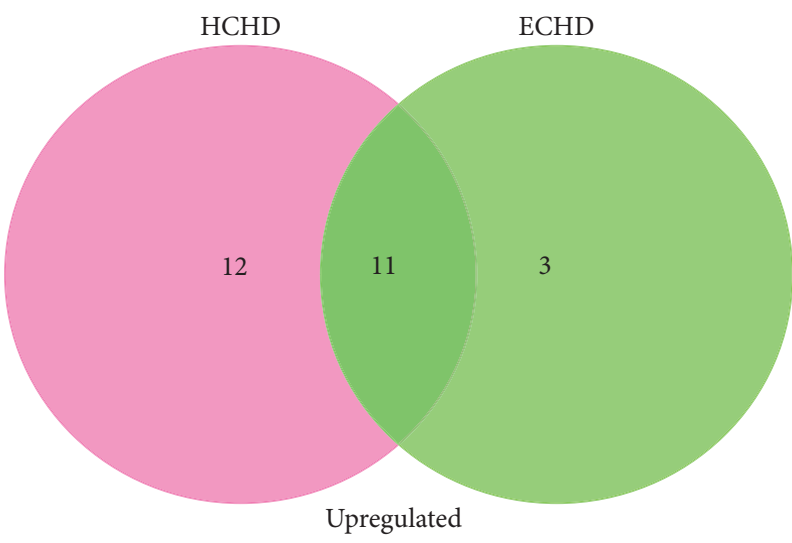

(a)

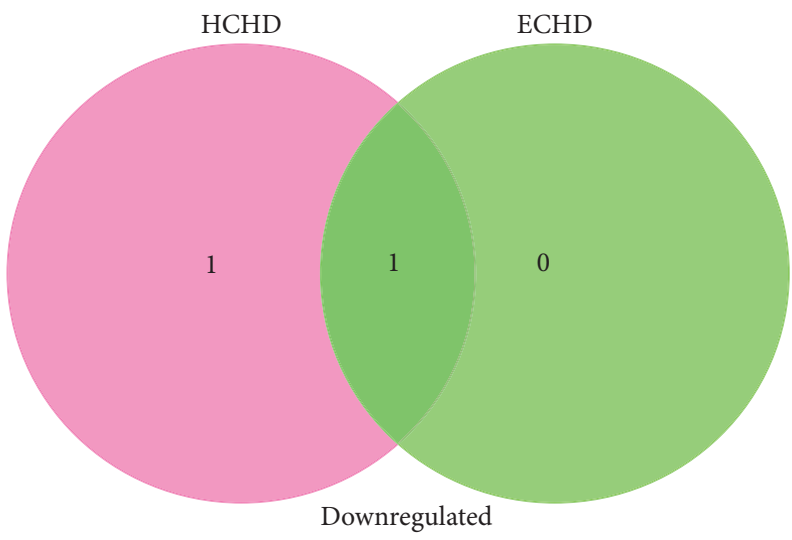

(b)

Figure 6: Venn diagrams of upregulated pathways (a) and downregulated pathways (b) from the target genes of differentially expressed miRNAs in HCHD and ECHD patients.

cardiovascular diseases, including acute myocardial infarction, heart failure, coronary artery disease, stroke, and hypertension [23]. MiRNA expression in HCHD and ECHD patients was explored by miRNA microarray analysis. According to the analysis, 1 specific miRNA was found in HCHD patients. Results indicated that hsa-miR-122-5p might be in connection with hyperglycemia in CHD patients. In the meantime, 6 specific miRNAs, including hsa-miR-451a, hsamiR-425-5p, and hsa-let-7b-5p, were only found in EHCD patients. Thirteen significantly upregulated miRNAs and 1 significantly downregulated miRNA are overlapped in the
HCHD and ECHD groups; the most significant upregulated and downregulated miRNAs are hsa-miR-16-5p and hsamiR-320a, respectively. This suggested that hsa-miR-16-5p and hsa-miR-320a might be closely connected to the occurrence of CHD. MiR-16 is related to cardiovascular diseases. Because miR-16 can downregulate expressions of VEGFR2 and FGFR1 to inhibit angiogenesis, its expression change also can induce myocardial cell hypertrophy [24]. MiR-16 has been reported to play a key role in regulation of insulin signaling through a reduction in TNF $\alpha$ and suppressor of cytokine signaling 3 (SOCS3) signaling and increase in 
TABLE 3: Specific pathways enriched by targets of differentially expressed miRNAs in HCHD group. (a) Top 10 significant upregulated pathways. (b) Significant downregulated pathway.

(a)

\begin{tabular}{|c|c|c|c|}
\hline KEGG pathway & FDR adjusted $p$ value & Genes & miRNAs \\
\hline TNF signaling pathway & $4.8 E-04$ & 30 & 12 \\
\hline Hippo signaling pathway & 0.0013 & 52 & 14 \\
\hline Signaling pathways regulating pluripotency of stem cells & 0.0017 & 49 & 12 \\
\hline Non-small cell lung cancer & 0.003 & 26 & 12 \\
\hline Epstein-Barr virus infection & 0.004 & 60 & 12 \\
\hline HTLV-I infection & 0.010 & 74 & 14 \\
\hline Small cell lung cancer & 0.011 & 33 & 13 \\
\hline PI3K-Akt signaling pathway & 0.015 & 93 & 14 \\
\hline Glioma & 0.022 & 27 & 13 \\
\hline TGF-beta signaling pathway & 0.024 & 32 & 11 \\
\hline
\end{tabular}

(b)

\begin{tabular}{lccc}
\hline KEGG pathway & FDR adjusted $p$ value & Genes & miRNAs \\
\hline Viral carcinogenesis & 0.019 & 20 & 2 \\
\hline
\end{tabular}

TABLE 4: Specific pathways enriched by targets of differentially expressed miRNAs in ECHD group.

\begin{tabular}{lccc}
\hline KEGG pathway & FDR adjusted $p$ value & Genes & miRNAs \\
\hline Ubiquitin mediated proteolysis & 0.005 & 50 & 15 \\
Wnt signaling pathway & 0.019 & 38 & 16 \\
Protein processing in endoplasmic reticulum & 0.033 & 56 & 19 \\
\hline
\end{tabular}

insulin-like growth factor binding protein-3 (IGFBP-3) levels to inhibit insulin resistance [25].

Target prediction by 2 databases (Tarbase and miRTarBase) identified large quantities of putative target genes for the differentially expressed miRNAs of HCHD and ECHD patients. And functional annotation by DAVID disclosed the biological properties and molecular functions of these target genes. The gene ontologies of HCHD and ECHD groups were compared to better understand the difference of biological property and molecular function of circulating miRNA profiles between HCHD and ECHD patients. The significantly upregulated and downregulated functional annotations of the miRNA targets in HCHD group included transcription, DNA-templated, protein polyubiquitination, regulation of cellular response to heat, nuclear membrane, nuclear pore, RISC complex, ubiquitin-protein ligase activity, ubiquitin-protein transferase activity, and double-stranded RNA binding, which suggested that hyperglycemia in $\mathrm{CHD}$ patients might relate to the exceptions of above biological processes and molecular functions.

In KEGG pathway analysis, a total of 25 significantly enriched pathways were found in HCHD group and a total of 15 significantly enriched pathways were found in ECHD group. TNF signaling pathway as the most significant upregulated pathway was found in HCHD group. It has been reported that TNF was shown to inhibit insulin signaling by serine phosphorylation of insulin receptors and protein phosphate PP-1 and by activation of protein tyrosine phosphatase SH-PTPase [26]. Therefore, it was speculated that the activation of TNF signaling pathway might be a reason for the occurrence of hyperglycemia in CHD patients. In addition, Hippo signaling pathway, signaling pathways regulating pluripotency of stem cells, PI3K-Akt signaling pathway, and TGF-beta signaling pathway were the top 10 significant upregulated pathways in HCHD group. PI3K/Akt signaling pathway is closely linked to plenty of biological processes such as proliferation, survival, migration, and cellular metabolic regulation and it is responsible for endothelial dysfunction and cell apoptosis caused by hyperglycemia [27]. PI3K/Akt signaling pathway which is thought to play an important role in insulin's metabolic function is a primary way to regulate the absorption and metabolism of glucose and balance the glucose level in blood [28]. Some studies have shown that insulin regulates blood glucose primarily through PI3K/Akt signaling pathway [29]. Wnt signaling pathway was found in ECHD group as a significant upregulated pathway. Wnt signaling pathway seems to be playing an important role in the occurrence of hypertension and diabetes, which has been demonstrated by clinical and in vitro studies [30]. Other studies exhibited an association between Wnt signaling pathway and the regulation of insulin secretion in $\beta$-cell [31]. It has been reported that activated Wnt signaling increases insulin secretion in isolated mouse islets and attenuated Wnt signaling decreases insulin content in both isolated 


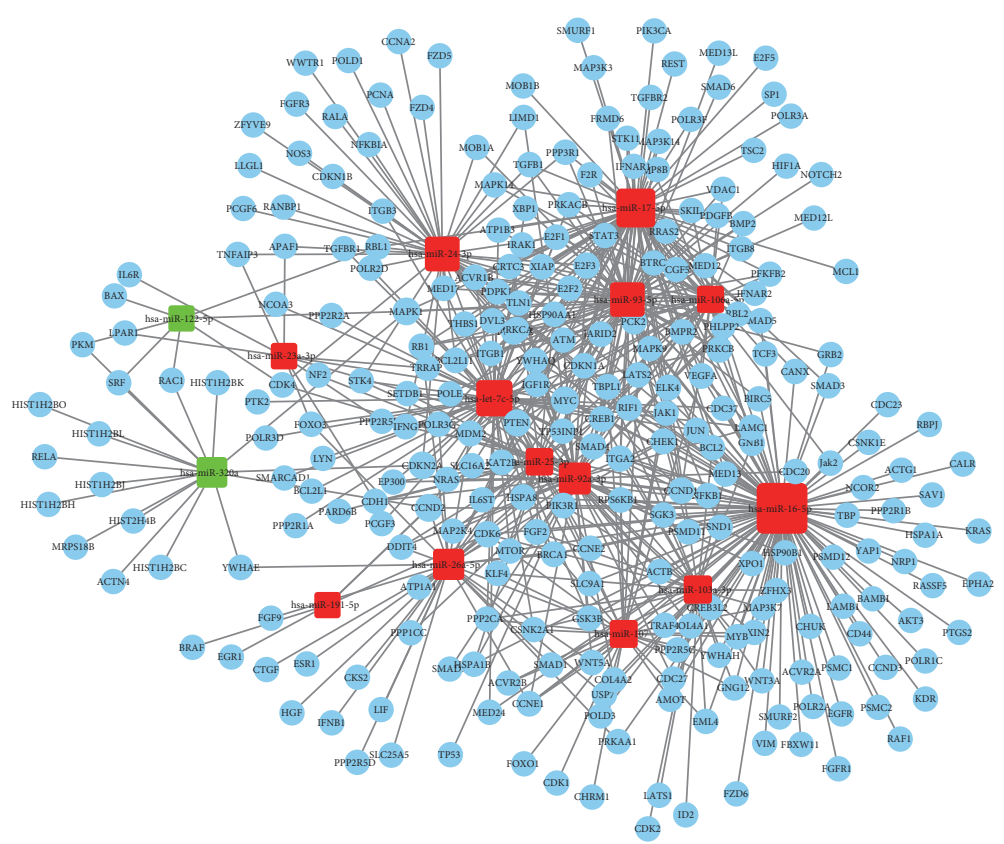

(a)

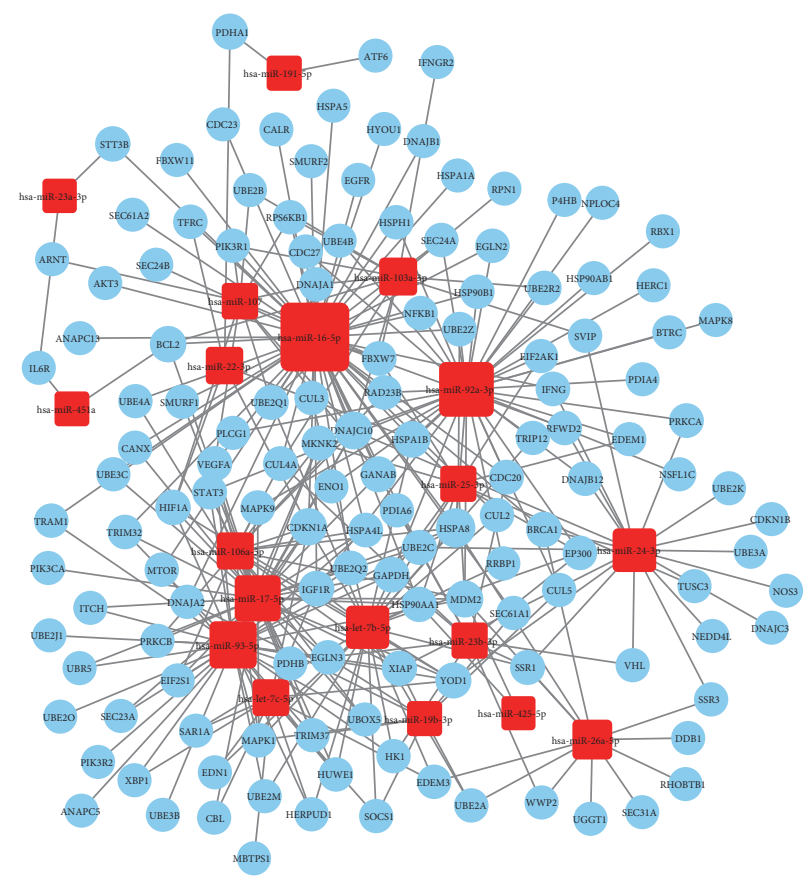

(b)

FIGURE 7: MiRNA-gene regulatory network diagram of HCHD (a) and ECHD (b). Red squares represent upregulated miRNAs and green squares represent downregulated miRNAs. Blue circles represent target genes. Black lines represent the regulatory relation between miRNAs and their target genes.

mouse and human islets [32]. Wnt signaling pathway was significant upregulated pathway in ECHD group. Therefore, the activation of Wnt signaling pathway might be the main reason that patients with $\mathrm{CHD}$ kept their blood glucose in the normal levels.

MiRNA-gene regulatory networks were constructed to investigate the function of differentially expressed miRNAs in
HCHD and ECHD groups. A detailed relationship between differentially expressed miRNAs and genes was found in HCHD groups. Results illustrated that upregulated miRNAs might play a more important role in the regulation of HCHD. Because hsa-miR-16-5p and hsa-miR-17-5p were core miRNAs in both HCHD and ECHD groups, these 2 miRNAs might play a crucial role in the occurrence and development 
of CHD. In addition, hsa-let-7c-5p and hsa-miR-24-3p might have the most important function for hyperglycemia in CHD patients and hsa-miR-92a-3p and hsa-miR-93-5p also were crucial in ECHD patients.

\section{Conclusions}

In the present study, we investigated the common and different mechanisms of miRNAs in HCHD and ECHD patients by comparing the biological properties, molecular functions, and enriched pathways of target genes of their differentially expressed circulating miRNAs. The biological characteristics of HCHD and ECHD and the molecular alterations in these two types of $\mathrm{CHD}$ were better understood through the study, which is conducive to explore novel therapies for future clinical applications to improve therapeutic efficacy and pertinence of treatment.

\section{Conflicts of Interest}

The authors declare that there are no conflicts of interest regarding the publication of this paper.

\section{Acknowledgments}

This work was supported financially by the National Basic Research Program of China (973 Program, 2015CB554400), Project Funded by China Postdoctoral Science Foundation (2017M611126), and National Major Research and Development Program Matching Project of Beijing (Z161100002616024).

\section{References}

[1] J. K. Fajar, "The $\beta$ fibrinogen gene G-455A polymorphism in Asian subjects with coronary heart disease: A meta analysis," Egyptian Journal of Medical Human Genetics, vol. 18, no. 1, pp. 19-28, 2017.

[2] D. Li, L. Zhao, J. Yu et al., "Lipoprotein-associated phospholipase A2 in coronary heart disease: review and meta-analysis," Clinica Chimica Acta, vol. 465, pp. 22-29, 2017.

[3] D. Wang, W. Li, X. Cui et al., "Sleep duration and risk of coronary heart disease: A systematic review and meta-analysis of prospective cohort studies," International Journal of Cardiology, vol. 219, pp. 231-239, 2016.

[4] Q. Chen, Y. Zhang, D. Ding et al., "Metabolic syndrome and its individual components with mortality among patients with coronary heart disease," International Journal of Cardiology, vol. 224, pp. 8-14, 2016.

[5] D. Fridman, A. N. Makaryus, J. N. Makaryus et al., "Diabetes and coronary artery disease-pathophysiologic insights and therapeutic implications," in Coronary Artery DiseaseAssessment, Surgery, Prevention, InTech, 2015.

[6] S. Benamer, I. Eljazwi, R. Mohamed, H. Masoud, M. Tuwati, and A. M. Elbarsha, "Association of hyperglycemia with in-hospital mortality and morbidity in Libyan patients with diabetes and acute coronary syndromes," Oman Medical Journal, vol. 30, no. 5, pp. 326-330, 2015.
[7] S. M. Welten, R. C. de Jong, A. Wezel et al., "Inhibition of 14q32 microRNA miR-495 reduces lesion formation, intimal hyperplasia and plasma cholesterol levels in experimental restenosis," Atherosclerosis, vol. 261, pp. 26-36, 2017.

[8] J. Shi, H. Liu, H. Wang, and X. Kong, "MicroRNA expression signature in degenerative aortic stenosis," BioMed Research International, vol. 2016, Article ID 4682172, 6 pages, 2016.

[9] Z.-M. Yang, L.-H. Chen, M. Hong et al., "Serum microRNA profiling and bioinformatics of patients with spleen-deficiency syndrome," Evidence-Based Complementary and Alternative Medicine, vol. 2016, Article ID 8726720, 9 pages, 2016.

[10] Y. Zhu, Y. Lin, W. Yan et al., "Novel biomarker micrornas for subtyping of acute coronary syndrome: a bioinformatics approach," BioMed Research International, vol. 2016, Article ID 4618323, 11 pages, 2016.

[11] E. K. Economou, E. Oikonomou, G. Siasos et al., "The role of microRNAs in coronary artery disease: from pathophysiology to diagnosis and treatment," Atherosclerosis, vol. 241, no. 2, pp. 624-633, 2015.

[12] T. P. Ram, I. Fomison-Nurse, S. Gandhi et al., "The diagnostic sensitivity of circulating cardio-enriched microRNAs is increased after normalization of high-density lipoprotein levels," International Journal of Cardiology, vol. 236, pp. 498500, 2017.

[13] R. Sodi, J. Eastwood, M. Caslake, C. J. Packard, and L. Denby, "Relationship between circulating microRNA-30c with totaland LDL-cholesterol, their circulatory transportation and effect of statins," Clinica Chimica Acta, vol. 466, pp. 13-19, 2017.

[14] M.-H. Mo, L. Chen, Y. Fu, W. Wang, and S. W. Fu, "Cell-free circulating miRNA biomarkers in cancer," Journal of Cancer, vol. 3, no. 1, pp. 432-448, 2012.

[15] A. Turchinovich, T. R. Samatov, A. G. Tonevitsky, and B. Burwinkel, "Circulating miRNAs: cell-cell communication function?” Frontiers in Genetics, vol. 4, article 119, 2013.

[16] R. Duttagupta, S. DiRienzo, R. Jiang et al., "Genome-wide maps of circulating miRNA biomarkers for Ulcerative Colitis," PLoS ONE, vol. 7, no. 2, Article ID e31241, 2012.

[17] I. S. Vlachos, M. D. Paraskevopoulou, D. Karagkouni et al., "DIANA-TarBase v7.0: indexing more than half a million experimentally supported miRNA:mRNA interactions," Nucleic Acids Research, vol. 43, no. 1, pp. D153-D159, 2015.

[18] C.-H. Chou, N.-W. Chang, S. Shrestha et al., "miRTarBase 2016: updates to the experimentally validated miRNA-target interactions database," Nucleic Acids Research, vol. 44, no. 1, pp. D239-D247, 2016.

[19] K. J. Gaulton, T. Nammo, L. Pasquali et al., "A map of open chromatin in human pancreatic islets," Nature Genetics, vol. 42, no. 3, pp. 255-259, 2010.

[20] J. Hou, J. Wang, C. Lin et al., "Circulating microRNA profiles differ between Qi-stagnation and Qi-deficiency in coronary heart disease patients with blood stasis syndrome," EvidenceBased Complementary and Alternative Medicine, vol. 2014, Article ID 926962, 9 pages, 2014.

[21] W. Zhang, Y. Xie, L. Xu et al., "Identification of microRNAs and their target genes explores miRNA-mediated regulatory network of cytoplasmic male sterility occurrence during anther development in radish (Raphanus sativus L.)," Frontiers in Plant Science, vol. 7, no. 2016, article 1054, 2016.

[22] X.-Y. Cao, J.-M. Lu, Z.-Q. Zhao et al., "MicroRNA biomarkers of Parkinson's disease in serum exosome-like microvesicles," Neuroscience Letters, vol. 644, pp. 94-99, 2017. 
[23] N. Li, J. Lian, S. Zhao et al., "Detection of differentially expressed microRNAs in rheumatic heart disease: mir-1183 and mir-1299 as potential diagnostic biomarkers," BioMed Research International, vol. 2015, Article ID 524519, 11 pages, 2015.

[24] C. Zheng, Q. Li, and W. Shen, "Roles of miR-16 in vascular endothelial injury in patients with coronary heart disease," Biomedical Research, vol. 28, no. 5, pp. 2337-2343, 2017.

[25] E.-A. Ye and J. J. Steinle, "miR-15b/16 protects primary human retinal microvascular endothelial cells against hyperglycemiainduced increases in tumor necrosis factor alpha and suppressor of cytokine signaling 3," Journal of Neuroinflammation, vol. 12, article 44, 8 pages, 2015.

[26] S. E. Borst, “The role of TNF- $\alpha$ in insulin resistance," Endocrine Journal, vol. 23, no. 2-3, pp. 177-182, 2004.

[27] W. Liu, B. Liu, S. Liu, J. Zhang, and S. Lin, "Sphingosine-1phosphate receptor 2 mediates endothelial cells dysfunction by PI3K-Akt pathway under high glucose condition," European Journal of Pharmacology, vol. 776, pp. 19-25, 2016.

[28] Y.-F. Gao, M.-N. Zhang, T.-X. Wang, T.-C. Wu, R.-D. Ai, and Z.-S. Zhang, "Hypoglycemic effect of D-chiro-inositol in type 2 diabetes mellitus rats through the PI3K/Akt signaling pathway," Molecular and Cellular Endocrinology, vol. 433, pp. 26-34, 2016.

[29] B. Dai, Q. Wu, C. Zeng et al., "The effect of Liuwei Dihuang decoction on PI3K/Akt signaling pathway in liver of type 2 diabetes mellitus (T2DM) rats with insulin resistance," Journal of Ethnopharmacology, vol. 192, pp. 382-389, 2016.

[30] P.-W. Cheng, Y.-Y. Chen, W.-H. Cheng et al., "Wnt signaling regulates blood pressure by Downregulating a GSK- $3 \beta$-mediated pathway to enhance insulin signaling in the central nervous system," Diabetes, vol. 64, no. 10, pp. 3413-3424, 2015.

[31] H. J. Welters and R. N. Kulkarni, "Wnt signaling: relevance to beta-cell biology and diabetes," Trends in Endocrinology \& Metabolism, vol. 19, no. 10, pp. 349-355, 2008.

[32] X. Wang, X. G. Lei, and J. Wang, "Malondialdehyde regulates glucose-stimulated insulin secretion in murine islets via TCF7L2-dependent Wnt signaling pathway," Molecular and Cellular Endocrinology, vol. 382, no. 1, pp. 8-16, 2014. 

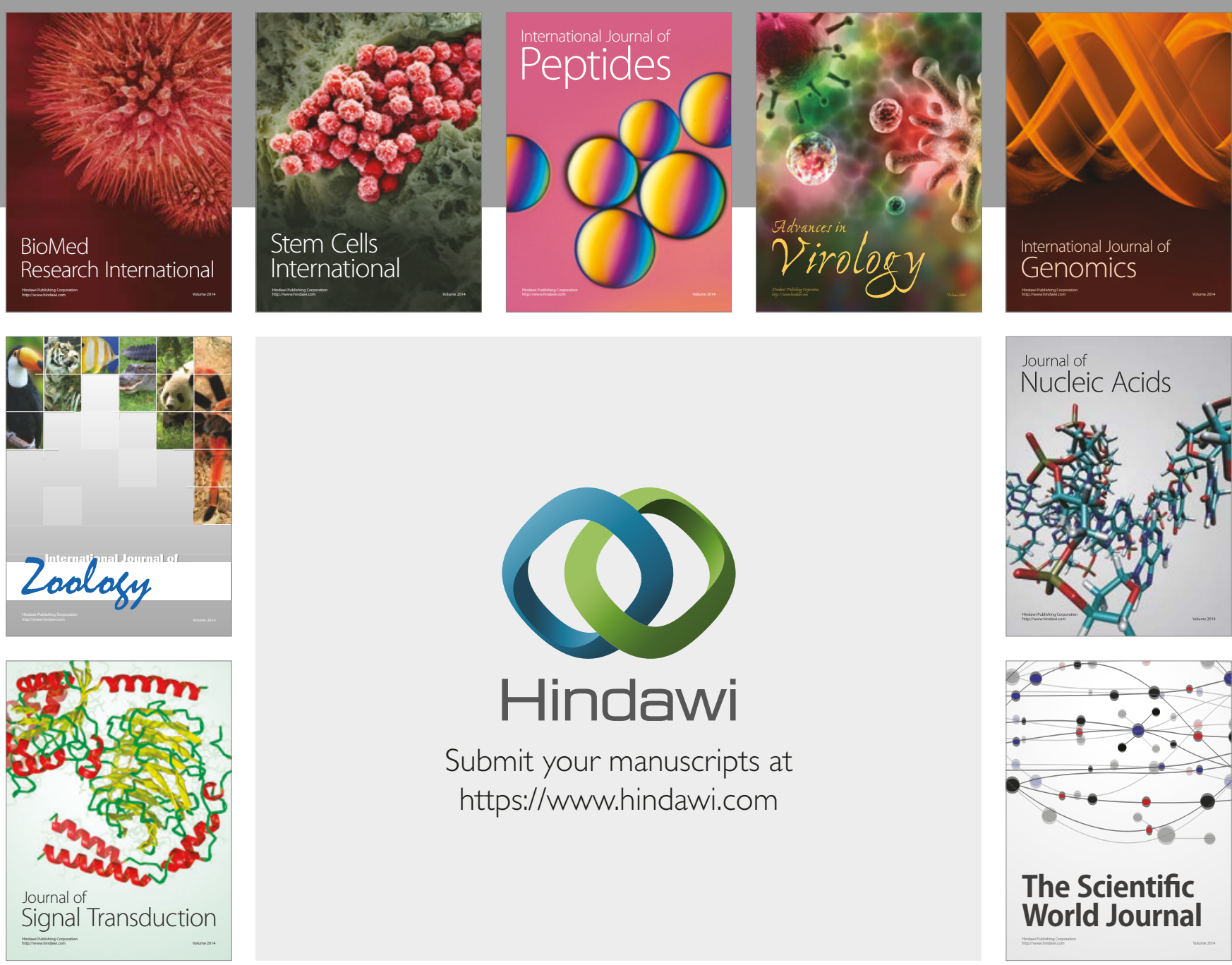

Submit your manuscripts at

https://www.hindawi.com
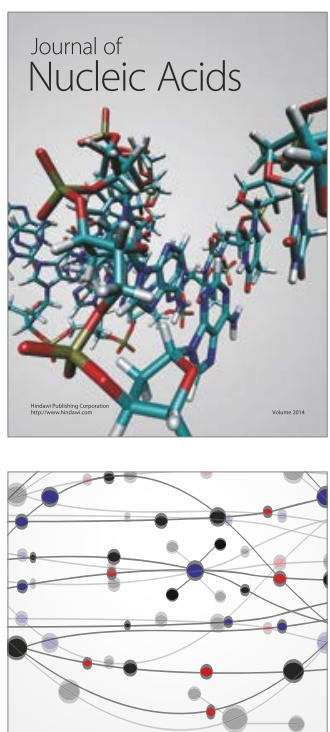

The Scientific World Journal

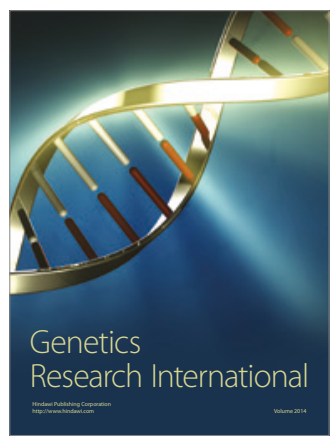

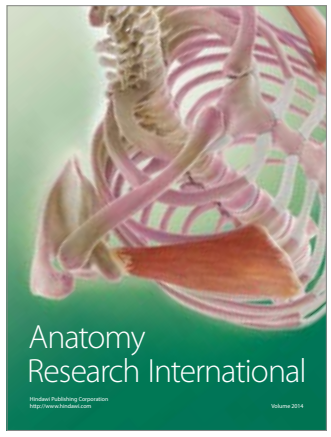

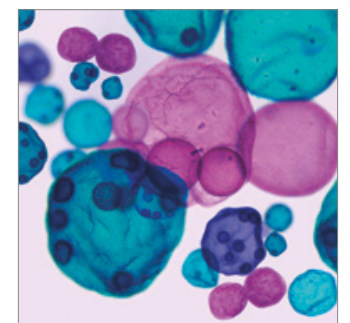

International Journal of Microbiology
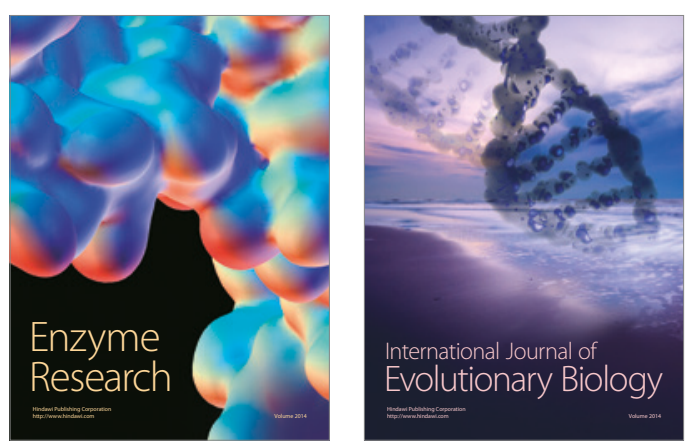
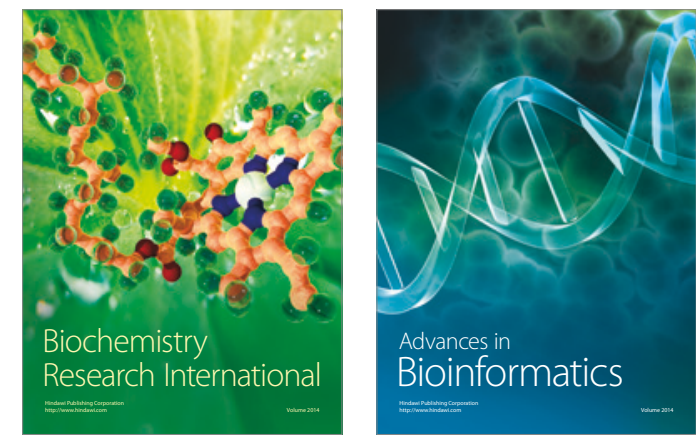

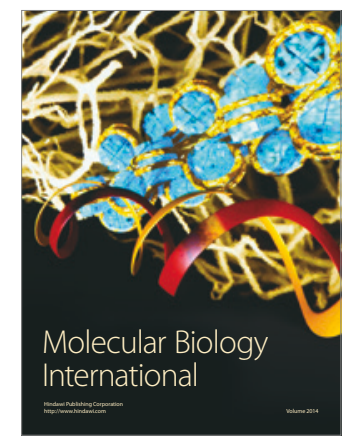

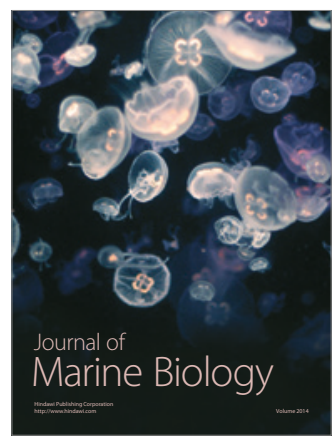

